\title{
Triglyceride-Glucose Index Predicts Adverse Events in Patients with Acute Coronary Syndrome: A Meta-Analysis of Cohort Studies
}

Authors

Juying Li, Ling Ren, Cheng Chang, Lin Luo

\author{
Affiliation \\ Department of Cardiovascular Medicine, The First People's \\ Hospital of Yibin City, Yibin, China
}

\author{
Key words \\ triglyceride-glucose index, insulin resistance, acute \\ coronary syndrome, major adverse cardiovascular events, \\ meta-analysis \\ received 16.03 .2021 \\ accepted after revision $\quad 12.05 .2021$ \\ published online 23.07 .2021 \\ Bibliography \\ Horm Metab Res 2021; 53: 594-601 \\ DOI $10.1055 / a-1518-7503$ \\ ISSN 0018-5043 \\ (c) 2021. Thieme. All rights reserved. \\ Georg Thieme Verlag KG, Rüdigerstraße 14, \\ 70469 Stuttgart, Germany \\ Correspondence \\ Dr. Juying Li \\ Department of Cardiovascular Medicine, The First People's \\ Hospital of Yibin City \\ No. 65 Wenxing Street \\ 644000 Yibin \\ Sichuan Province \\ China \\ Tel.: + 868318212087 , Fax: + 868318212087 \\ juying_li@21cn.com
}

\section{ABSTRACT}

The triglyceride-glucose (TyG) index, a recently proposed indicator for insulin resistance, has been related with cardiovascular risks. We aimed to summarize the association between TyG index and incidence of major adverse cardiovascular events (MACEs) in patients with acute coronary syndrome (ACS). Cohort studies demonstrating the association between TyG index and incidence of MACEs in ACS patients with multivariate adjusted analyses were identified by search of PubMed, Embase, and Web of Science databases. A random-effekt model incorporating the heterogeneity was applied to pool the results. Eight cohort studies with 19611 participants were included. Results showed that compared to those with the lowest category of TyG index, ACS patients with the highest category of TyG index were independently associated with higher risk of MACEs [risk ratio (RR): 1.94, $95 \%$ confidence interval $(\mathrm{Cl})$ : $\left.1.47-2.56, \mathrm{I}^{2}=85 \%, \mathrm{p}<0.001\right)$. Subgroup analyses showed consistent results in patients with ST-segment elevated myocardial infarction or non-ST segment elevated ACS, in patients with or without diabetes, and in patients after percutaneous coronary intervention. Results were consistent in studies with TyG index analyzed as continuous variable (RR for per standard deviation increment of TyG index: $1.59,95 \% \mathrm{Cl}$ : 1.38-1.83, $\left.\mathrm{I}^{2}=24 \%, \mathrm{p}<0.001\right)$. In conclusion, higher TyG index may be independently associated with higher incidence of MACEs in patients with ACS.

\section{Introduction}

Acute coronary syndrome (ACS) is a category of clinically severe coronary artery disease (CAD) characterized by the acute rupture of vulnerable atherosclerotic plaques and subsequent total occlusion of the coronary arteries [1,2]. Clinically, ACS could be further classified as ST segment elevated myocardial infarction (STEMI) and non-ST segment elevated ACS (NSTE-ACS) [1]. Despite of continuing advances in the treatment of ACS, particularly of the revascularization therapy such as percutaneous coronary intervention $(\mathrm{PCI})$, patients with ACS remain of poor prognosis [3,4]. Accordingly, identification of predictors for prognosis in ACS patients has becoming more and more important for improving the risk strati- fication of the patients and development of novel treatment strategies $[5,6]$.

Accumulating evidence from experimental and epidemiological studies suggests that insulin resistance plays key roles in the pathogenesis and progression of CAD in both the diabetic and non-diabetic patients [7, 8]. However, previous studies evaluating the potential prognostic role of insulin resistance in ACS patients are rare, possibly due to the lack of reliable and convenient surrogate indicator of insulin resistance [9]. Indeed, the "gold standard" indicator of insulin sensitivity is the hyperinsulinemic-euglycemic clamp test [10], while the application of the index is limited by the time consuming and expensive nature of the method [11]. Recent- 
ly, the triglyceride-glucose (TyG) index, which is derived from fasting blood glucose and triglyceride, is suggested as a reliable and easily obtained indicator of insulin resistance [12]. A higher TyG index has been associated with the risk of CAD in general population $[13,14]$. Besides, TyG index has also been related with the severity of coronary calcification $[15,16]$. However, the association between TyG index and the incidence of major adverse cardiovascular events (MACEs) in patients with ACS remains not fully determined [17-24]. Therefore, in this study, we aimed to summarize the potential independent association between TyG index and subsequent incidence of MACEs in ACS patients by performing a meta-analysis of previous published cohort studies. Besides, influences of subtype of ACS and diabetic status of the patients on the above association were also explored in subgroup analyses.

\section{Materials and Methods}

The MOOSE (Meta-analysis of Observational Studies in Epidemiology) [25] Statement and Cochrane's Handbook [26] were followed in the design, performing and reporting of the meta-analysis.

\section{Literature search}

Electronic databases including PubMed, Embase, and Web of Science were searched with the combination of the following terms: (1) "TyG index" OR "triglyceride-glucose index" OR "triglyceride and glucose index" OR "triglyceride glucose index" OR "triacylglycerol glucose index"; and (2) "coronary artery disease" OR "angina" OR "myocardial infarction" OR "acute coronary syndrome" OR "percutaneous coronary intervention" OR "major adverse cardiovascular events" OR "CAD" OR "STEMI" OR "NSTEMI" OR "ACS" OR "AMI" OR "PCl". Filters of human were applied and only studies published in English or Chinese were considered. References lists of related original and review articles were manually searched for potential eligible studies. The final literature search was performed on January 18, 2021.

\section{Study selection}

Studies that fulfilled all of the following criteria were included: (1) cohort studies published as full-length articles; (2) included patients with ACS at baseline; (3) evaluated the association between TyG index and the incidence of MACEs during follow-up; and (4) reported the relative risk for the association after adjustment of potential confounding factors. TyG index was calculated as $\ln [\mathrm{TG}$ (mg/ $\mathrm{dl}) \times$ FPG $(\mathrm{mg} / \mathrm{dl}) / 2$ ] [27]. The definition of MACEs was in accordance with the included studies, which was defined as composite outcomes of all-cause death, non-fatal myocardial infarction, non-fatal stroke, revascularization, and cardiac rehospitalization. Cross-sectional studies, reviews, preclinical studies, and studies irrelevant to the aim of the meta-analysis were excluded.

\section{Data extracting and quality evaluation}

Literature search, data extraction, and quality assessment of the included studies were performed by 2 authors independently according to the predefined criteria. Discrepancies were resolved by consensus. The extracted data were: (1) name of first author, publication year, and country; (2) study design characteristics; (3) patient characteristics, including the diagnosis, sample size, age, sex, and proportions of patients with diabetes; (4) patterns for TyG index analysis; (5) follow-up durations; (6) numbers of patients with subsequent incidence of MACEs; and (7) confounding factors adjusted in the multivariate analyses. The quality of each study was evaluated using the Newcastle-Ottawa Scale [28]. This scale judges the quality of cohort studies with 3 domains: selection of the study groups; the comparability of the groups; and the ascertainment of the outcome of interest. The quality score of NOS ranges from 1 to 9 stars in total.

\section{Statistical analyses}

Risk ratios (RRs) and their corresponding $95 \%$ confidence intervals (Cls) were applied as the general measure for the association between TyG index and risk of MACEs in ACS patients. For studies with TyG index analyzed as categorized variables, RRs of MACEs for participants with the highest TyG index level compared to those with the lowest TyG index level were extracted. For studies with TyG index analyzed as continuous variables, RRs of MACEs incidence per 1-standard deviation (SD) increment of TyG index was extracted. Data of RRs and their stand errors (SEs) were calculated from $95 \%$ Cls or p-values, and were logarithmically transformed for the stabilization of the variance and normalization of the distribution [26]. To indicate a potential independent association between TyG index and subsequent incidence of MACEs in patients with ACS, only RR data with the most adequately adjusted model of multivariate analysis was extracted and combined. The Cochrane's $Q$ test was used to evaluate the heterogeneity among the include cohort studies, as well as the estimation of $\mathrm{I}^{2}$ statistic [29]. If $\mathrm{I}^{2}>50 \%$, a significant heterogeneity was considered. We used a random-effekt model to synthesize the RR data because this model is considered as a more generalized method which could incorporate the potential heterogeneity among the included studies [26]. Sensitivity analyses, which exclude one individual study at a time, were performed to test the stability of the results [30]. Predefined subgroup analyses were performed to evaluate the influences of subtype of ACS and diabetic status of the patients on the association. Further meta-analysis restricted to patients with after $\mathrm{PCl}$ was also performed. A p-value $<0.05$ indicates statistical significance. The potential publication bias was assessed by visual inspection of the funnel plots for symmetry, as well as the Egger's regression asymmetry test [31]. RevMan (Version 5.1; Cochrane Collaboration, Oxford, UK) and STATA (Version 12.0) software were used to perform the meta-analysis and statistics.

\section{Results}

\section{Literature search}

- Figure 1 shows the process of database search. Briefly, 597 articles were obtained via initial literature search of the databases after excluding of the duplications. Among them, 567 were excluded through screening of the titles and abstracts for irrelevance. Subsequently, 30 articles underwent full-text review. Of these, 22 were further excluded for the reasons listed in $>$ Fig. 1. Finally, 8 cohort studies were obtained for the meta-analysis [17-24]. 


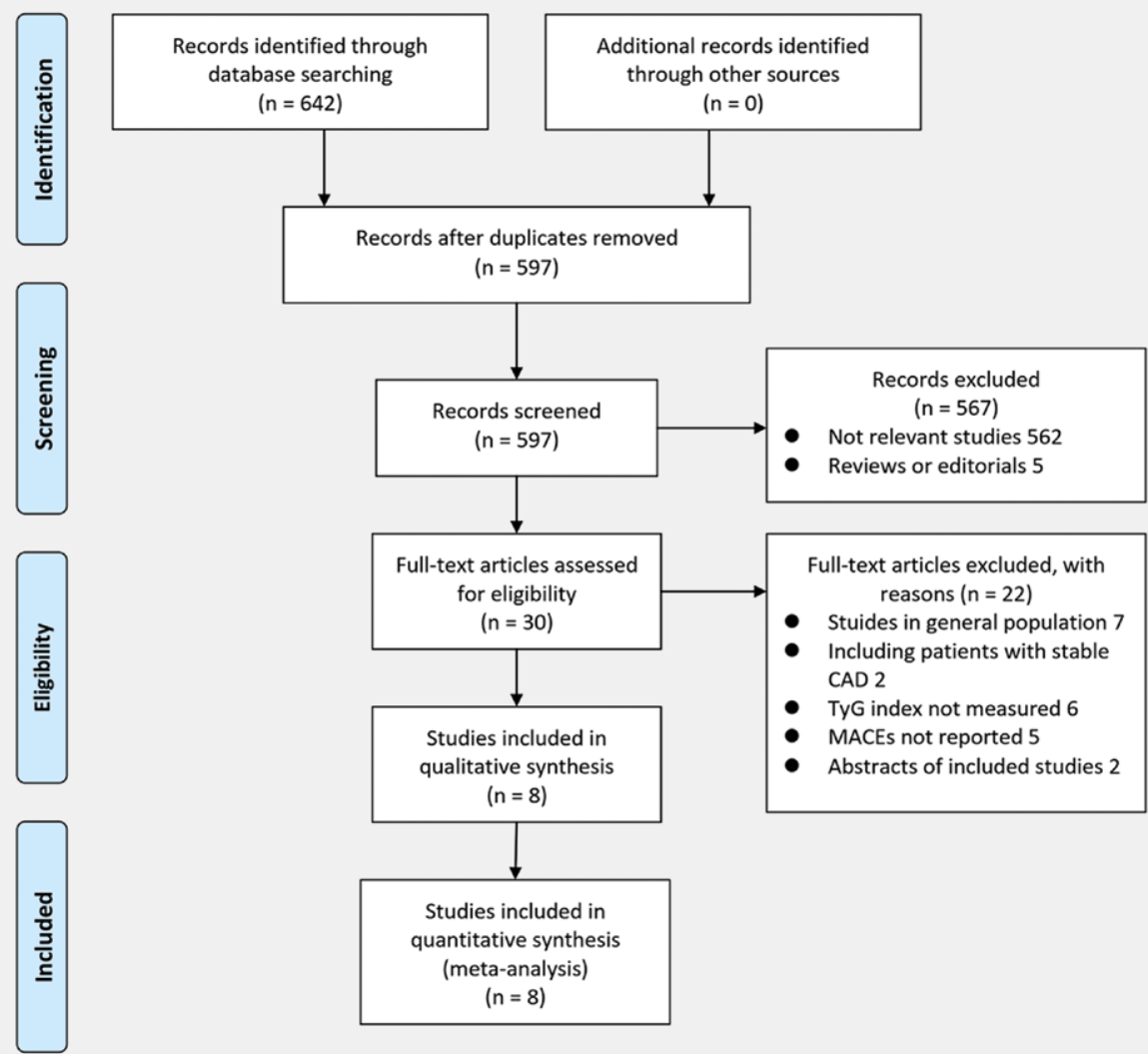

- Fig. 1 Flowchart of database search and study identification.

\section{Study characteristics and quality evaluation}

Eight cohort studies including 19611 participants were included in the meta-analysis. The detailed study characteristics were shown in $>$ Table 1. All of the available studies were performed in China. Two of them were prospective $[18,20]$, and the remaining 6 were retrospective $[17,19,21-24]$. The sample sizes of the included studies varied between 438 and 9285 . Mean ages of the included patients with ACS varied from 59 to 67 years. All of the included studies provided data of TyG index analyzed as a categorized variable, while 4 studies also analyzed as a continuous variable $[20,21,23,24]$. With a follow-up duration of $1 \sim 3$ years, 2762 patients had subsequent MACEs. Age, sex, BMI, smoking status, blood pressure, serum total cholesterol or low-density lipoprotein cholesterol, diabetic status, features of coronary lesions, revascularization therapy, and concurrent medications were adjusted to a varying degree when the association between TyG index and MACEs were studied. The NOS scores of the included studies were 8 9, indicating good study quality ( $\triangleright$ Table 2 ).

\section{TyG index and the incidence of MACEs}

ACS patients with the highest category of TyG index were independently associated with higher risk of MACEs (RR: $1.94,95 \% \mathrm{Cl}$ : 1.47-2.56, $\mathrm{I}^{2}=85 \%, \mathrm{p}<0.001$; > Fig. 2a). Consistent results were obtained for meta-analysis with TyG index analyzed as continuous variables (4 studies [20,21, 23, 24], RR for 1-SD increment of TyG index: 1.59 , $95 \% \mathrm{Cl}: 1.38-1.83, \mathrm{I}^{2}=24 \%, \mathrm{p}<0.001$; $>$ Fig. 2b). Re- sults of sensitivity analyses by excluding one study at a time showed similar results. For meta-analysis of studies with TyG index analyzed as categorized variables, subgroup analyses showed consistent results in patients with STEMI or NSTE-ACS, and in patients with or without diabetes (both $p$ for subgroup difference >0.05; $>$ Fig. 3a,b). Meta-analysis restricted to patients after $\mathrm{PCl}$ also showed similar results (RR: 2.14, $95 \% \mathrm{Cl}: 1.66-2.75, \mathrm{I}^{2}=73 \%$, $\mathrm{p}<0.001$; - Fig. 3c).

\section{Publication bias}

The funnel plots regarding the meta-analysis with TyG index analyzed as a categorized variable were shown in $>$ Fig. 4 . The funnel plots were symmetry on visual inspection, suggesting low risk of publication bias. Egger's regression tests showed consistent results $(p=0.197)$. Publication bias for the meta-analysis with TyG index analyzed as a continuous variable was difficult to estimate since limited studies were included.

\section{Discussion}

In this meta-analysis of relevant cohort studies, we found that higher TyG index was independently associated with increased incidence of subsequent MACEs in patients with ACS. Consistent results were obtained for meta-analyses of studies of TyG index analyzed as categorized variables and continuous variables. Besides, sensitivity analyses by omitting one study at a time confirmed the 


\begin{tabular}{|c|c|c|c|c|c|c|c|c|c|c|c|}
\hline & 气̆ & & $\infty$ & $a$ & $a$ & $\infty$ & $\infty$ & $\infty$ & $\infty$ & $\infty$ & \\
\hline & 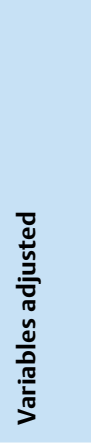 & & 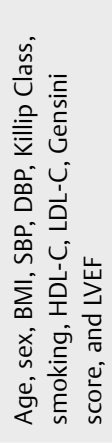 & 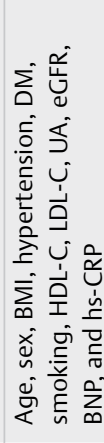 & 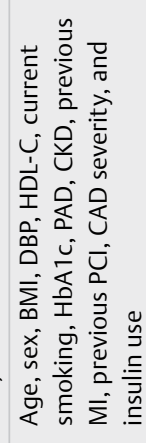 & 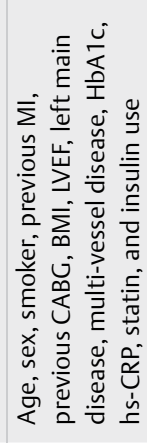 & 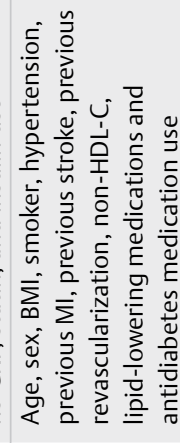 & 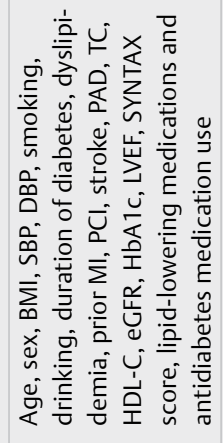 & 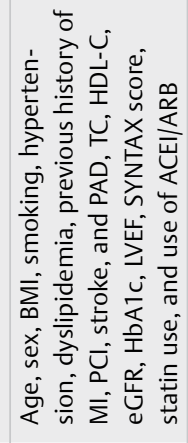 & 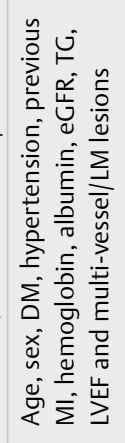 & 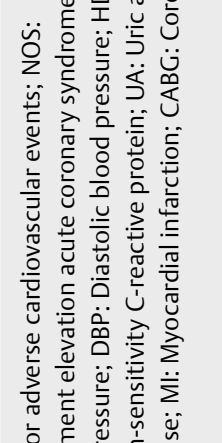 \\
\hline & 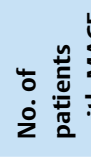 & & $\stackrel{n}{n}$ & $\stackrel{\infty}{\curvearrowright}$ & $\stackrel{\infty}{\infty}$ & $\stackrel{\infty}{\infty}$ & $\stackrel{\infty}{\stackrel{\infty}{+}}$ & $\stackrel{\infty}{\circ}$ & $\frac{6}{m}$ & 㞻 & 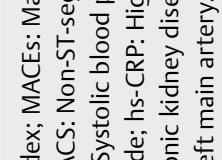 \\
\hline & 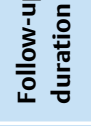 & 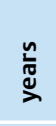 & $\stackrel{\circ}{r}$ & $\stackrel{\circ}{-}$ & 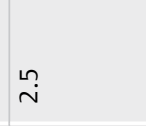 & $\dot{m}$ & $\stackrel{\stackrel{n}{\longrightarrow}}{\leftarrow}$ & $\dot{m}$ & $\stackrel{\circ}{+}$ & $\stackrel{\infty}{i}$ & 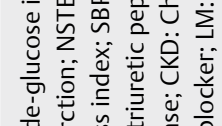 \\
\hline & $\begin{array}{l}\frac{n}{n} \\
\frac{n}{\pi} \\
\frac{\pi}{\pi} \\
\stackrel{5}{\gtrless}\end{array}$ & & $\begin{array}{l}\bar{o} \\
\dot{\sigma}\end{array}$ & 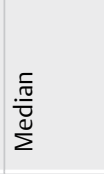 & 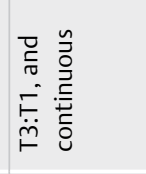 & 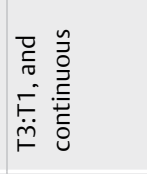 & $\underset{\ddot{m}}{F}$ & 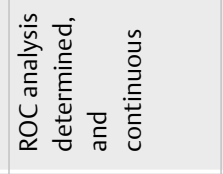 & 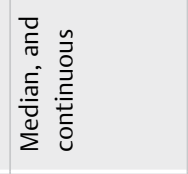 & 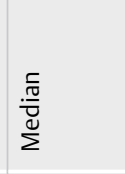 & 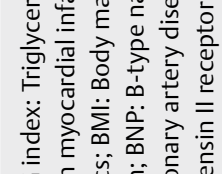 \\
\hline & $\bar{\Xi}$ & ๙゚ & $\stackrel{\circ}{\circ}$ & 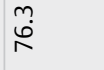 & 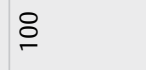 & $\stackrel{\text { tin }}{\text { in }}$ & 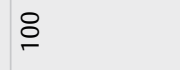 & $\stackrel{\circ}{\circ}$ & $\stackrel{8}{-}$ & ৪ & 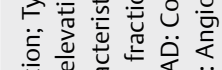 \\
\hline & $\sum_{0}$ & ๙゚ & $\stackrel{\dot{\sim}}{\sim}$ & $\stackrel{\sim}{m}$ & 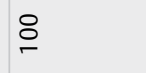 & 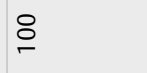 & 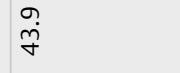 & $\stackrel{-}{\circ}$ & 0 & $m_{m}^{\infty}$ & 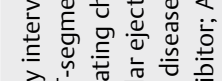 \\
\hline & $\frac{\frac{\varrho}{\pi N}}{2}$ & ஃீ & Бे & $\stackrel{0}{6}$ & 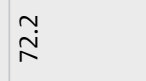 & นิ่ & $\stackrel{m}{\stackrel{m}{n}}$ & $\infty_{\infty}^{m}$ & $\stackrel{m}{i}$ & 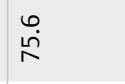 & \\
\hline & 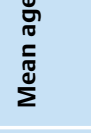 & $\stackrel{\coprod}{\beth}$ & ชิ & $\widetilde{6}$ & $\frac{0}{\dot{\sigma}}$ & 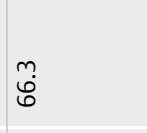 & ले & 8 & ต่ & 8 & 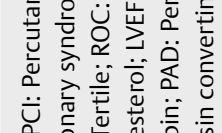 \\
\hline & 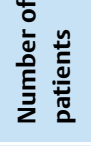 & & ᄋి & $\stackrel{\infty}{\dddot{\gamma}}$ & $\frac{0}{1}$ & $\stackrel{N}{N}$ & $\stackrel{\infty}{\sim}$ & న্ & $\stackrel{\check{n}}{\sim}$ & $\frac{\infty}{m}$ & 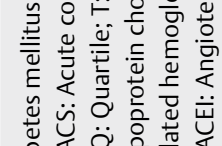 \\
\hline 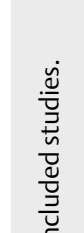 & 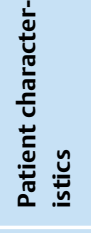 & & 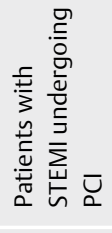 & 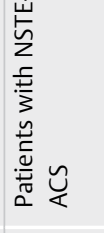 & 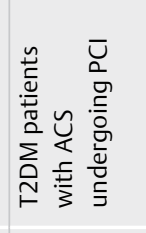 & 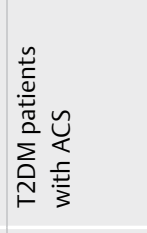 & 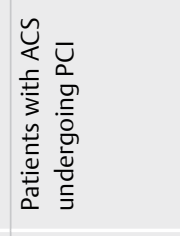 & 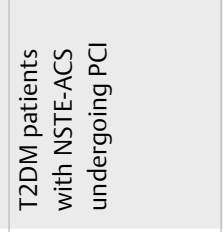 & 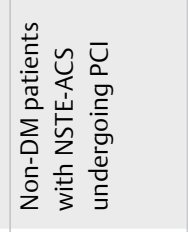 & 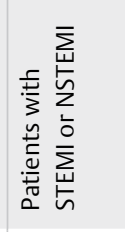 & 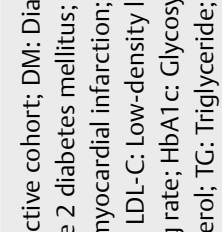 \\
\hline 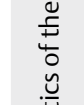 & 点 & & $\approx$ & 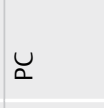 & $a$ & $\approx$ & $\propto$ & $\approx$ & $\approx$ & $\approx$ & 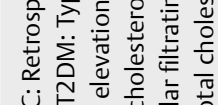 \\
\hline 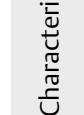 & 站 & & 昙 & : & 窇 & 薹 & $\stackrel{\frac{\pi}{\tilde{E}}}{\tilde{U}}$ & 参 & 毫 & . & 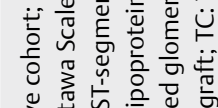 \\
\hline $\begin{array}{l}\overline{\frac{5}{0}} \\
\frac{0}{\pi} \\
\frac{\pi}{\Delta}\end{array}$ & 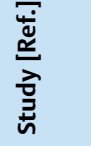 & & $\begin{array}{l}\stackrel{0}{o} \\
\stackrel{N}{\Xi} \\
\stackrel{\Xi}{\Xi}\end{array}$ & $\begin{array}{l}\frac{\infty}{2} \\
i \\
0 \\
\sum^{\infty} \infty \\
\sum^{\infty}\end{array}$ & 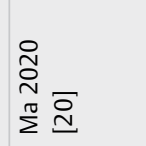 & 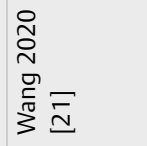 & 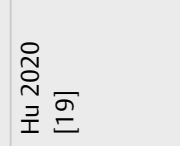 & 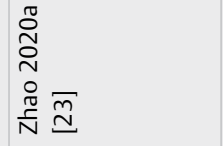 & 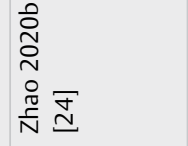 & 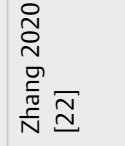 & 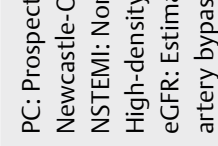 \\
\hline
\end{tabular}


- Table 2 Details of study quality evaluation via the Newcastle-Ottawa Scale.

\begin{tabular}{|c|c|c|c|c|c|c|c|c|c|c|}
\hline Study & $\begin{array}{l}\text { Represent- } \\
\text { ativeness } \\
\text { of the } \\
\text { exposed } \\
\text { cohort }\end{array}$ & $\begin{array}{l}\text { Selection } \\
\text { of the non- } \\
\text { exposed } \\
\text { cohort }\end{array}$ & $\begin{array}{l}\text { Ascertain- } \\
\text { ment of } \\
\text { exposure }\end{array}$ & $\begin{array}{l}\text { Outcome } \\
\text { not } \\
\text { present at } \\
\text { baseline }\end{array}$ & $\begin{array}{l}\text { Control } \\
\text { for age }\end{array}$ & $\begin{array}{l}\text { Control for } \\
\text { other } \\
\text { confound- } \\
\text { ing factors }\end{array}$ & $\begin{array}{l}\text { Assess- } \\
\text { ment of } \\
\text { outcome }\end{array}$ & $\begin{array}{l}\text { Enough long } \\
\text { follow-up } \\
\text { duration }\end{array}$ & $\begin{array}{l}\text { Adequacy of } \\
\text { follow-up of } \\
\text { cohorts }\end{array}$ & Total \\
\hline Luo 2019 & 0 & 1 & 1 & 1 & 1 & 1 & 1 & 1 & 1 & 8 \\
\hline Mao 2019 & 1 & 1 & 1 & 1 & 1 & 1 & 1 & 1 & 1 & 9 \\
\hline Ma 2020 & 1 & 1 & 1 & 1 & 1 & 1 & 1 & 1 & 1 & 9 \\
\hline Wang 2020 & 0 & 1 & 1 & 1 & 1 & 1 & 1 & 1 & 1 & 8 \\
\hline Hu 2020 & 0 & 1 & 1 & 1 & 1 & 1 & 1 & 1 & 1 & 8 \\
\hline Zhao 2020a & 0 & 1 & 1 & 1 & 1 & 1 & 1 & 1 & 1 & 8 \\
\hline Zhao 2020b & 0 & 1 & 1 & 1 & 1 & 1 & 1 & 1 & 1 & 8 \\
\hline Zhang 2020 & 0 & 1 & 1 & 1 & 1 & 1 & 1 & 1 & 1 & 8 \\
\hline
\end{tabular}

a

\begin{tabular}{|c|c|c|c|c|}
\hline Study or Subgroup & log[Risk Ratio] & SE & Weight & $\begin{array}{l}\text { Risk Ratio } \\
\text { IV. Random. } 95 \% \mathrm{CI}\end{array}$ \\
\hline Zhang 2020 & 0.173953 & 0.08511 & $14.5 \%$ & $1.19[1.01,1.41]$ \\
\hline Luo 2019 & 0.424614 & 0.18423 & $12.2 \%$ & $1.53[1.07,2.19]$ \\
\hline Wang 2020 & 0.429832 & 0.15336 & $13.0 \%$ & $1.54[1.14,2.08]$ \\
\hline Mao 2019 & 0.630207 & 0.25917 & $10.1 \%$ & $1.88[1.13,3.12]$ \\
\hline Zhao 2020b & 0.735728 & 0.13555 & $13.4 \%$ & $2.09[1.60,2.72]$ \\
\hline Ma 2020 & 0.765468 & 0.20529 & $11.6 \%$ & $2.15[1.44,3.22]$ \\
\hline Hu 2020 & 0.858662 & 0.13338 & $13.5 \%$ & $2.36[1.82,3.07]$ \\
\hline Zhao 2020a & 1.401675 & 0.20239 & $11.7 \%$ & $4.06[2.73,6.04]$ \\
\hline Total $(95 \% \mathrm{Cl})$ & & & $100.0 \%$ & $1.94[1.47,2.56]$ \\
\hline \multicolumn{5}{|c|}{ Heterogeneity: $\mathrm{Tau}^{2}=0.13 ; \mathrm{Chi}^{2}=46.64, \mathrm{df}=7(\mathrm{p}<0.00001) ; \mathrm{I}^{2}=85 \%$} \\
\hline
\end{tabular}

b

Risk Ratio

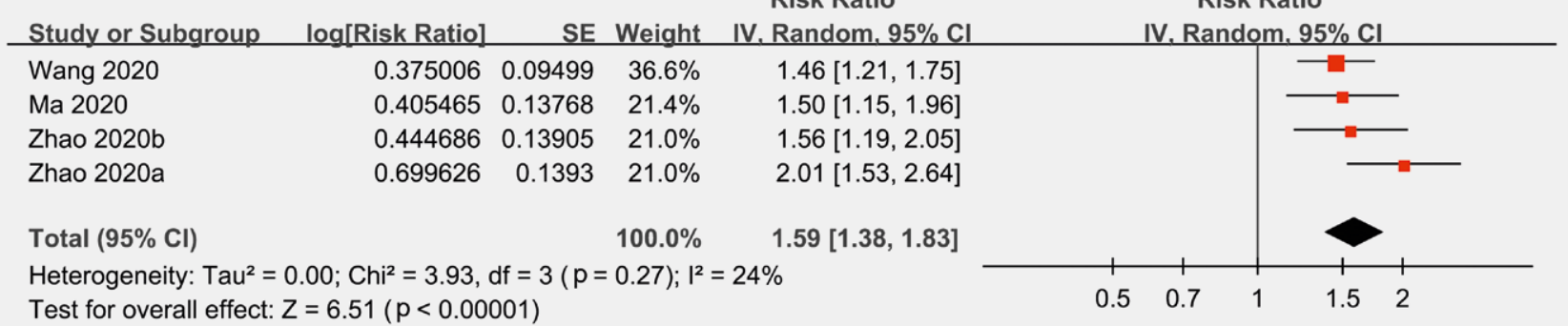

- Fig. 2 Forest plots for the meta-analysis of the association between TyG index and risk of subsequent MACEs in ACS patients; a: meta-analysis with TyG index analyzed as categorized variables; and b: meta-analysis with TyG index analyzed as continuous variables.

robustness of the results. In addition, further subgroup analyses showed consistent results in patients with STEMI or NSTE-ACS, with or without diabetes, and in those after $\mathrm{PCl}$. Collectively, these findings suggested that higher TyG index may be an independent predictor of MACEs in patients with ACS.

To the best of our knowledge, this is the first meta-analysis which summarized the association between TyG index and risk of MACEs in ACS patients. Our study has several strengths. First, only cohort studies were included, which avoided the potential bias inherited in the cross-sectional studies, such as patient selection bias and recall bias. In addition, only studies with multivariate analyses were included, which enabled us to indicate a potential independent association between TyG index and subsequent risk of MACEs. Moreover, consistent results were obtained for meta-analyses with TyG index analyzed categorized or continuous variables, and for sensitivity and subgroup analyses, which all suggested the robustness and stability of the results. Taken together, these findings support that insulin resistance as evaluated by TyG index could be a prognostic factor for patients with ACS. These findings were consistent with the results of previous studies, which showed that a 
a

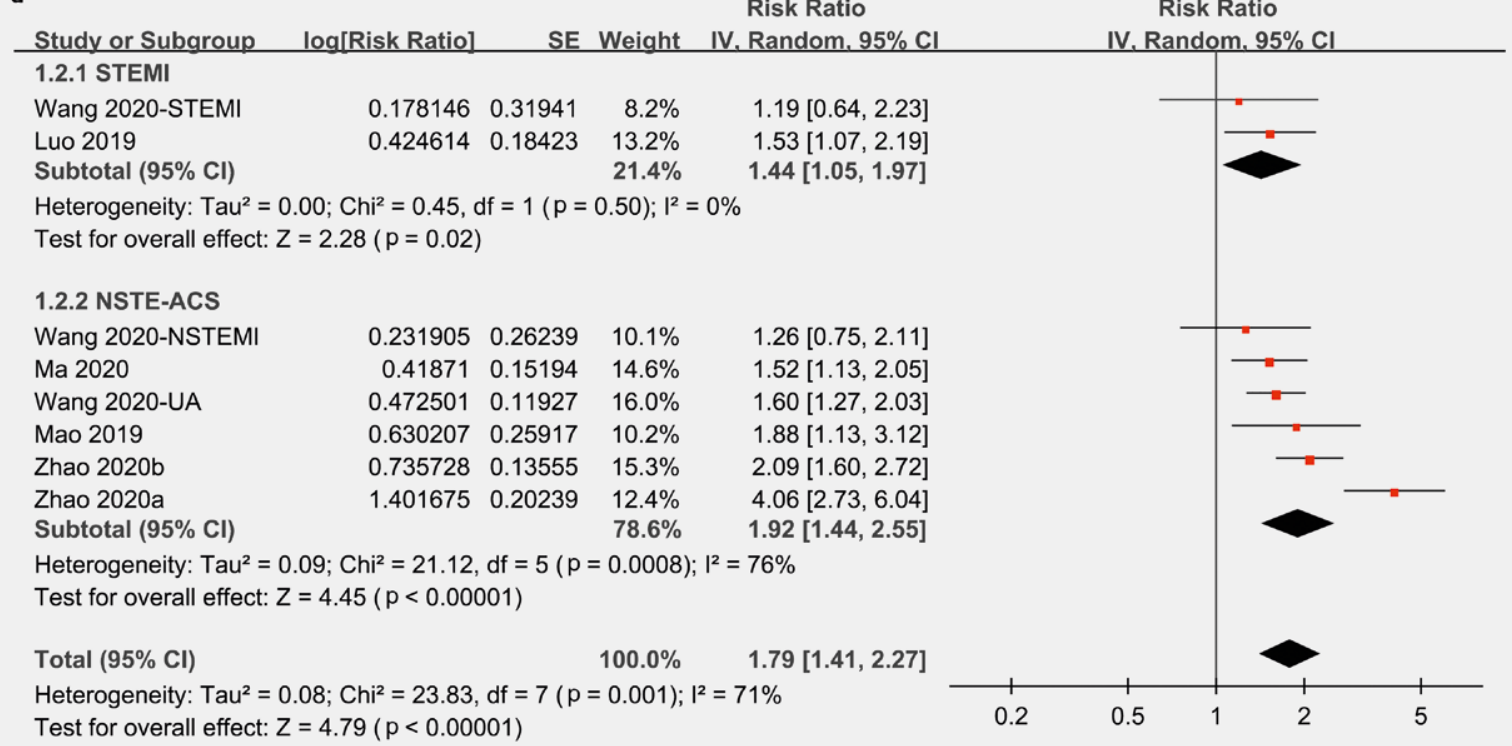

Test for subaroun differences: $\mathrm{Chi}^{2}=1.76 . \mathrm{df}=1(\mathrm{p}=0.18) . \mathrm{I}^{2}=43.3 \%$

b

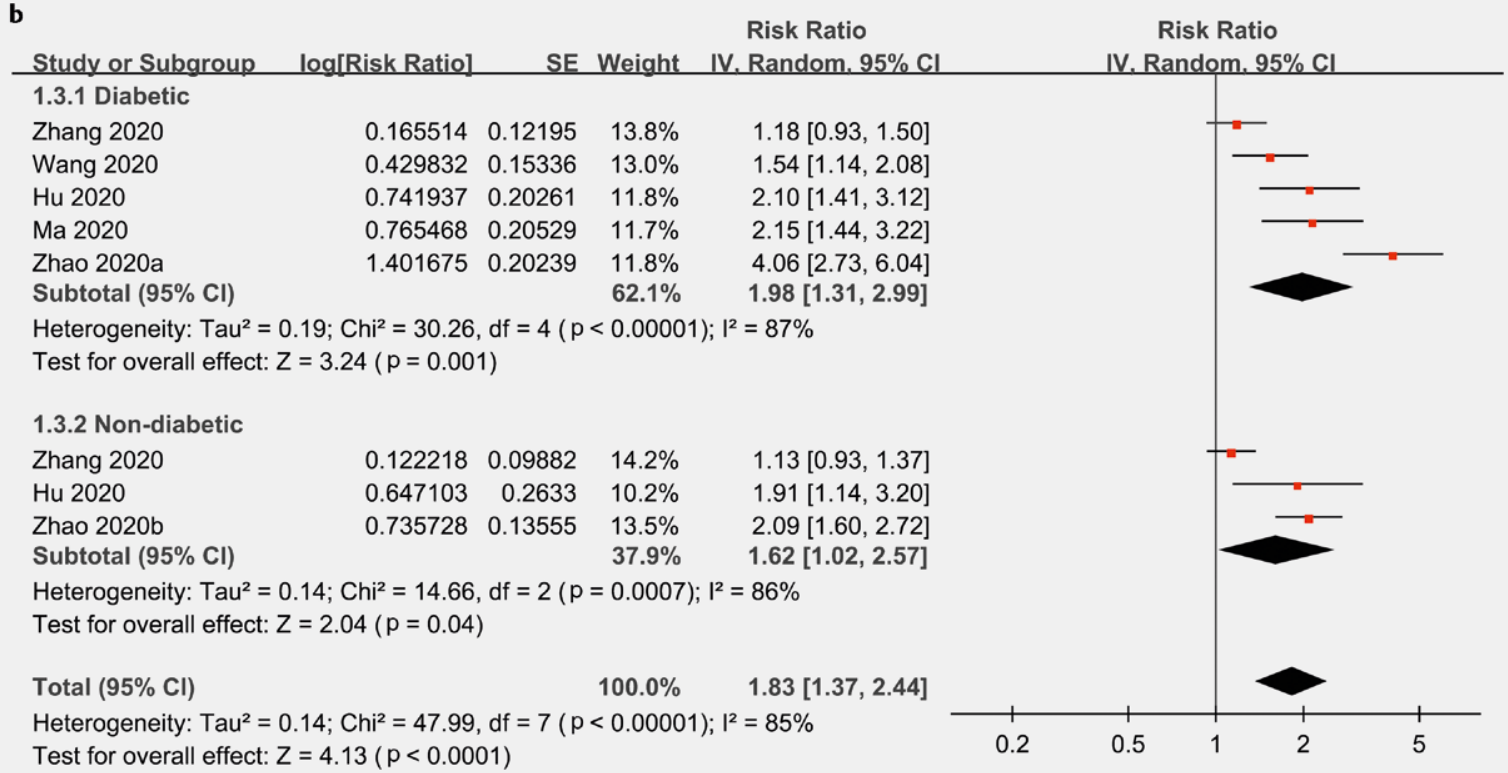

Test for subaroun differences: $\mathrm{Chi}^{2}=0.41 . \mathrm{df}=1(\mathrm{p}=0.52) . \mathrm{I}^{2}=0 \%$

c

- Fig. 3 Subgroup analyses for the meta-analysis with TyG index analyzed as categorized variables; a: subgroup analysis according to the subtype of ACS; b: subgroup analysis according to the diabetic status of the patients; and c: meta-analysis limited to patients after PCI. 


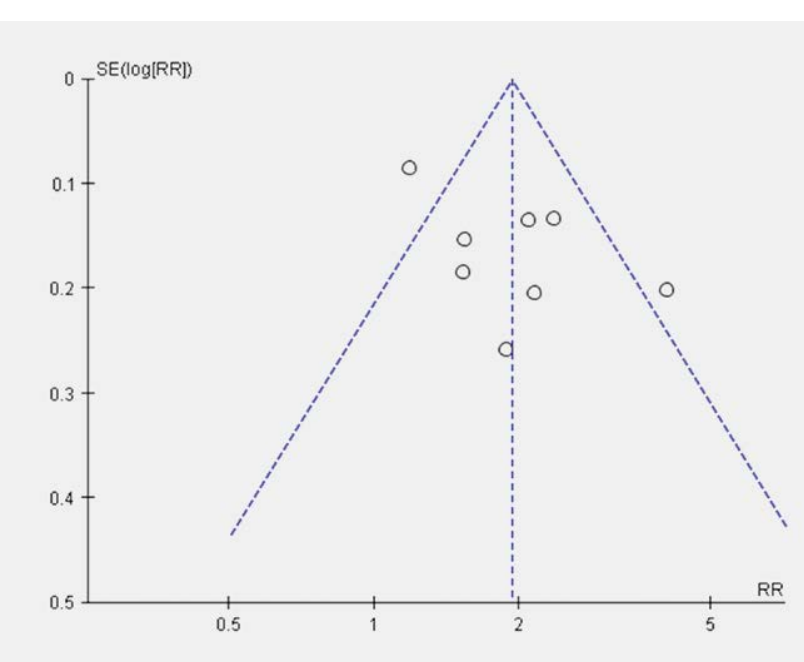

- Fig. 4 Funnel plots for the publication bias underlying the meta-analysis with TyG index analyzed as categorized variables.

higher TyG index was related with the severity of coronary lesions in patients with CAD [12]. An early study including 2840 participants who underwent coronary computed tomography angiography showed that the TyG index was independently associated with the presence and severity of CAD due to an increased risk for calcified or mixed plaque [32]. Moreover, subsequent follow-up study showed that TyG index is an independent predictor of coronary artery calcification progression in adult Koreans [16]. Besides, in patients with NSTE-ACS, higher TyG index was independently related to severer coronary lesions as evidenced by higher SYNTAX score [18]. These findings may partly explain the potential association between higher TyG index and increased risk of subsequent MACEs in ACS patients. Pathophysiologically, insulin resistance has been associated with low-grade but persistent inflammatory response [33], which has been associated with the rupture of atherosclerotic plaques in ACS [34]. Besides, higher TyG index has also be related to endothelial dysfunction and impaired autonomic function $[35,36]$, which were both considered as predictors of poor clinical outcomes in ACS patients [37]. Future studies are needed to determine the exact molecular mechanisms underlying the potential association between TyG index and risk of MACEs in ACS patients.

Measuring insulin resistance with TyG index was easy, inexpensive, and fast based on routine blood biochemical tests. Besides, TyG index was shown to with a high sensitivity $(96.5 \%)$ and specificity $(85.0 \%)$ for evaluation of insulin resistance compared to the hyperinsulinemic-euglycemic clamp test [38]. In addition, TyG index was shown to be better than homeostatic model assessment for measuring insulin resistance (HOMA-IR) [39], which supported the use of TyG index as a potential component for risk stratification of ACS patients. Future studies are needed to determine the predictive efficacy of adding TyG index to established risk scores in ACS patients. Comparisons for the predictive efficacies between TyG index and HOMA-IR for MACEs in patients with ACS are also important for real-world clinical practice, and future studies are also warranted.
This meta-analysis also has some limitations. Firstly, limited studies were available for the meta-analysis, all performed in China, and significant heterogeneity was detected. The influences of other study characteristics on the outcome should be further investigated, such as the ethnicity and treatments for ACS. Moreover, it remains unknown if the association between TyG index and risk of MACEs was linear. Besides, the optimal cutoff value of TyG index as a prognostic factor for ACS patients could not been determined based on the results of current meta-analysis and previous studies. Studies are needed in the future to address this issue. Also, although studies with multivariate analyses were included, residual confounding factors may still exist which may affekt the association between TyG index and MACEs in ACS patients. Particularly, difference in dietary and nutritional factors, and use of glycemic and lipids-lowering medications may affekt TyG index, which may further influence the association between TyG index and subsequent incidence of MACEs [40]. However, we were unable to determine the potential influences of these factors on the association between TyG index and MACEs since these variables were rarely reported among the included studies. Finally, TyG index was measured for once among the included studies. The stability of the index, changes of TyG index during the hospitalization and after discharge and their associations with subsequent incidence of MACEs in ACS patients remain to be determined.

In conclusion, current evidence from cohort studies suggests that higher TyG index may be an independent predictor of subsequent risk of MACEs in patients with ACS. Since measuring insulin resistance with TyG index was reliable, easy, fast, and inexpensive, future studies are needed to determine whether incorporation of TyG index into current ACS risk stratification scores could improve their predictive efficacies.

\section{Conflict of Interest}

The authors declare that they have no conflict of interest.

\section{References}

[1] Jia S, Liu Y, Yuan J. Evidence in Guidelines for Treatment of Coronary Artery Disease. Adv Exp Med Biol 2020; 1177: 37-73

[2] Daghash H, Lim Abdullah K, Ismail MD. The effekt of acute coronary syndrome care pathways on in-hospital patients: A systematic review. J Eval Clin Pract 2020; 26: 1280-1291

[3] Virani SS, Alonso A, Aparicio H] et al. Heart Disease and Stroke Statistics-2021 Update: A Report From the American Heart Association. Circulation 2021; CIR0000000000000950

[4] Neumann FJ, Sousa-Uva M, Ahlsson A et al. 2018; ESC/EACTS Guidelines on myocardial revascularization. Eur Heart J 2019: 87-165

[5] Aarts GWA, Mol JQ, Camaro C et al. Recent developments in diagnosis and risk stratification of non-ST-elevation acute coronary syndrome. Neth Heart J 2020; 28: 88-92

[6] Chan Pin Yin D, Azzahhafi J, James S. Risk Assessment Using Risk Scores in Patients with Acute Coronary Syndrome. J Clin Med 2020 Sep 21; 9(9): 3039. doi:10.3390/jcm9093039. 
[7] Adeva-Andany MM, Martinez-Rodriguez J, Gonzalez-Lucan M et al. Insulin resistance is a cardiovascular risk factor in humans. Diabetes Metab Syndr 2019; 13: 1449-1455

[8] Patel TP, Rawal K, Bagchi AK et al. Insulin resistance: an additional risk factor in the pathogenesis of cardiovascular disease in type 2 diabetes. Heart Fail Rev 2016; 21: 11-23

[9] Gerstein HC, Ferrannini E, Riddle MC et al. Insulin resistance and cardiovascular outcomes in the ORIGIN trial. Diabetes Obes Metab 2018; 20: 564-570

[10] Rebelos E, Honka MJ. PREDIM index: a useful tool for the application of the euglycemic hyperinsulinemic clamp. J Endocrinol Invest 2021; 44: 631-634

[11] Tura A, Chemello G, Szendroedi ] et al. Prediction of clamp-derived insulin sensitivity from the oral glucose insulin sensitivity index. Diabetologia 2018; 61: 1135-1141

[12] Alizargar ], Bai CH, Hsieh NC et al. Use of the triglyceride-glucose index (TyG) in cardiovascular disease patients. Cardiovasc Diabetol 2020; 19 : 8

[13] Hong S, Han K, Park CY. The triglyceride glucose index is a simple and low-cost marker associated with atherosclerotic cardiovascular disease: a population-based study. BMC Med 2020; 18: 361

[14] da Silva A, Caldas APS, Hermsdorff HHM et al. Triglyceride-glucose index is associated with symptomatic coronary artery disease in patients in secondary care. Cardiovasc Diabetol 2019; 18: 89

[15] Won KB, Park EJ, Han D et al. Triglyceride glucose index is an independent predictor for the progression of coronary artery calcification in the absence of heavy coronary artery calcification at baseline. Cardiovasc Diabetol 2020; 19: 34

[16] Park K, Ahn CW, Lee SB et al. Elevated TyG Index Predicts Progression of Coronary Artery Calcification. Diabetes Care 2019; 42: 1569-1573

[17] Luo E, Wang D, Yan G et al. High triglyceride-glucose index is associated with poor prognosis in patients with acute ST-elevation myocardial infarction after percutaneous coronary intervention. Cardiovasc Diabetol 2019; 18: 150

[18] Mao Q, Zhou D, Li Y et al. The Triglyceride-Glukose Index Predicts Coronary Artery Disease Severity and Cardiovascular Outcomes in Patients with Non-ST-Segment Elevation Acute Coronary Syndrome. Dis Markers 2019; 6891537

[19] Hu C, Zhang J, Liu J et al. Discordance between the triglyceride glucose index and fasting plasma glucose or $\mathrm{HbA} 1 \mathrm{C}$ in patients with acute coronary syndrome undergoing percutaneous coronary intervention predicts cardiovascular events: A cohort study from China. Cardiovasc Diabetol 2020; 19: 116

[20] Ma X, Dong L, Shao Q et al. Triglyceride glucose index for predicting cardiovascular outcomes after percutaneous coronary intervention in patients with type 2 diabetes mellitus and acute coronary syndrome. Cardiovasc Diabetol 2020; 19: 31

[21] Wang L, Cong HL, Zhang JX et al. Triglyceride-glucose index predicts adverse cardiovascular events in patients with diabetes and acute coronary syndrome. Cardiovasc Diabetol 2020; 19: 80

[22] Zhang Y, Ding X, Hua B et al. High triglyceride-glucose index is associated with adverse cardiovascular outcomes in patients with acute myocardial infarction. Nutr Metab Cardiovasc Dis 2020; 30: 2351-2362

[23] Zhao Q, Zhang TY, Cheng Y] et al. Impacts of triglyceride-glucose index on prognosis of patients with type 2 diabetes mellitus and nonST-segment elevation acute coronary syndrome: results from an observational cohort study in China. Cardiovasc Diabetol 2020; 19: 108

[24] Zhao Q, Zhang TY, Cheng Y] et al. Triglyceride-Glukose Index as a Surrogate Marker of Insulin Resistance for Predicting Cardiovascular Outcomes in Nondiabetic Patients with Non-ST-Segment Elevation Acute Coronary Syndrome Undergoing Percutaneous Coronary Intervention. J Atheroscler Thromb 2020; doi:10.5551/jat.59840. Online ahead of print
[25] Stroup DF, Berlin JA, Morton SC et al. Meta-analysis of observational studies in epidemiology: a proposal for reporting. Meta-analysis Of Observational Studies in Epidemiology (MOOSE) group. JAMA 2000; 283: 2008-2012

[26] Higgins ], Green S. Cochrane Handbook for Systematic Reviews of Interventions Version 5.1.0. The Cochrane Collaboration. 2011; www.cochranehandbook.org

[27] Simental-Mendia LE, Rodriguez-Moran M, Guerrero-Romero F. The product of fasting glucose and triglycerides as surrogate for identifying insulin resistance in apparently healthy subjects. Metab Syndr Relat Disord 2008; 6: 299-304

[28] Wells GA, Shea B, O'Connell D et al. The Newcastle-Ottawa Scale (NOS) for assessing the quality of nonrandomised studies in meta-analyses. 2010; http://www.ohri.ca/programs/clinical_epidemiology/oxford.asp

[29] Higgins JP, Thompson SG. Quantifying heterogeneity in a meta-analysis. Stat Med 2002; 21: 1539-1558

[30] Patsopoulos NA, Evangelou E, loannidis JP. Sensitivity of between-study heterogeneity in meta-analysis: proposed metrics and empirical evaluation. Int J Epidemiol 2008; 37: 1148-1157

[31] Egger M, Davey Smith G, Schneider M et al. Bias in meta-analysis detected by a simple, graphical test. BMJ 1997; 315: 629-634

[32] Won KB, Kim YS, Lee BK et al. The relationship of insulin resistance estimated by triglyceride glucose index and coronary plaque characteristics. Medicine (Baltimore) 2018; 97: e10726

[33] Matulewicz N, Karczewska-Kupczewska M. Insulin resistance and chronic inflammation. Postepy Hig Med Dosw (Online) 2016; 70: 1245-1258

[34] Bentzon JF, Otsuka F, Virmani R et al. Mechanisms of plaque formation and rupture. Circ Res 2014; 114: 1852-1866

[35] Ormazabal V, Nair S, Elfeky O et al. Association between insulin resistance and the development of cardiovascular disease. Cardiovasc Diabetol 2018; 17: 122

[36] Poon AK, Whitsel EA, Heiss $G$ et al. Insulin resistance and reduced cardiac autonomic function in older adults: the Atherosclerosis Risk in Communities study. BMC Cardiovasc Disord 2020; 20: 217

[37] Cieslik-Guerra UI, Fila M, Kaminski M et al. Correlation between the activity of the autonomic nervous system and endothelial function in patients with acute coronary syndrome. Pol Arch Med Wewn 2014; 124: 509-515

[38] Guerrero-Romero F, Simental-Mendia LE, Gonzalez-Ortiz M et al. The product of triglycerides and glucose, a simple measure of insulin sensitivity. Comparison with the euglycemic-hyperinsulinemic clamp. J Clin Endocrinol Metab 2010; 95: 3347-3351

[39] Vasques AC, Novaes FS, de Oliveira Mda S et al. TyG index performs better than HOMA in a Brazilian population: a hyperglycemic clamp validated study. Diabetes Res Clin Pract 2011; 93: e98-e100

[40] Shahavandi M, Djafari F, Shahinfar $\mathrm{H}$ et al. The association of plant-based dietary patterns with visceral adiposity, lipid accumulation product, and triglyceride-glucose index in Iranian adults. Complement Ther Med 2020; 53: 102531

\section{Notice}

This article was changed according to the erratum on September 28, 2021.

\section{Erratum}

There was a misspelling in the title of the above mentioned article, the correct title is: Triglyceride-Glucose Index Predicts Adverse Events in Patients with Acute Coronary Syndrome: A Meta-Analysis of Cohort Studies. 